\title{
QoS-Aware Multi-tier Location Managements for Integrated WLAN/UMTS Networks
}

\author{
Yun Won Chung \\ School of Electronic Engineering, Soongsil University, \\ 511 Sangdo-Dong, Dongjak-Gu, Seoul 156-743, Korea \\ ywchung@ssu.ac.kr
}

\begin{abstract}
This paper addresses quality of service (QoS)-aware multi-tier location managements for integrated WLAN/UMTS networks. Single registration (SR) and multiple registration (MR) schemes are introduced and improved to support both voice and data services efficiently. The performance of the improved SR and MR schemes is analyzed based on mobility and traffic characteristics of mobile users. The results show that there is tradeoff between improved SR and MR schemes and thus, an appropriate scheme should be selected based on users' mobility and traffic characteristics. The analytical methodology developed in this paper can be extended to analyze the performance of general heterogeneous networks with multiple wireless access systems, which will be realized in fourth generation $(4 \mathrm{G})$ networks.
\end{abstract}

Keywords: Location management, multi-tier, registration, quality of service.

\section{Introduction}

Many wireless systems, such as cellular, wireless LAN (WLAN), mobile Ad hoc, sensor, digital video broadcasting (DVB), and satellite systems have been developed independently in order to meet the specific need of each system, such as cellular for high mobility and WLAN for high speed data service with low cost. Since these systems are also complimentary to each other in nature, the integration of these heterogeneous wireless systems is able to meet the ever increasing demand for anywhere, anytime, high speed data service.

The integration of heterogeneous wireless access systems based on common all-IP packet core is one of the most common visions for future mobile systems (e.g., $4 \mathrm{G}$ or beyond $3 \mathrm{G}$ ) in mobile research community [1]. The $4 \mathrm{G}$ system supports global roaming to mobile users and satisfies their diverse service demands in all aspects, e.g., quality of service (QoS) and cost, via the best available access system.

In such a $4 \mathrm{G}$ system, global roaming should be supported always, even when mobile users move across heterogeneous wireless access systems, which makes the location management of $4 \mathrm{G}$ system very challenging. In location management, a mobile terminal (MT) notifies the network of its current registration area (RA) and it is stored in location register. Then, the location information is retrieved in call delivery process and paging is performed to find the cell of the MT within the RA.

In $4 \mathrm{G}$ system with various access systems, if there is no co-operative location management scheme, location updates are performed in all available systems independently, 
which results in high signalling load in access networks and high power consumption in MT due to multiple location updates sent by one MT. Reversely, paging is also simultaneously performed in all access systems redundantly and scarce radio resources are wasted.

In order to solve these problems, a new location management scheme is needed for heterogeneous network with multiple access systems. In this paper, we are primarily concerned with location management for integrated WLAN/UMTS networks as a preliminary study for location management for general heterogeneous network with multiple access systems because UMTS will be the main cellular system in a near future and WLAN has been widely deployed in hot spot areas.

There have been many studies on the location management for WLAN/UMTS networks. In 3GPP, feasibility on the interworking between UMTS and WLAN has been studied, where the interworking requirements and interworking scenarios are defined [2], [3]. In [4], a possible architecture for integrating UMTS and 802.11 WLAN is proposed, which allows an MT to maintain data connection through WLAN and voice connection through UMTS in parallel. In [5], mobility management procedures within and between UMTS and 802.11 WLAN for various integration scenarios are addressed and interaction between various networks elements and new procedures for seamless mobility are considered. In [6], dormant mode operation support for WLAN-UMTS roaming for dual-mode users is addressed.

These studies, however, only deal with interworking procedures and implementation aspects, but do not analyze the performance of the integrated WLAN/UMTS location management. A few studies have been conducted on the performance analysis of multi-tier location managements supporting voice service only [7] - [10]. In [7], single registration (SR) and multiple registration (MR) schemes with multi-tier home location register (MHLR) are proposed. In SR, an MT is allowed to register with MHLR on only one tier out of tiers available, where high tier corresponds to Advanced Mobile Phone System (AMPS) and low tier corresponds to Personal Access Communication System (PACS). Since low tier provides voice service with lower cost, priority is always given to low tier if both tiers are available. On the contrary, an MT is allowed to register with MHLR on multiple tiers at the same time in MR in order to remove frequent tier switchings in SR. From [7], it can be concluded that there is tradeoff between SR and MR schemes depending on the mobility and traffic characteristics. In [8] - [10], the performance of SR scheme and single-tier scheme has been analyzed in detail considering low-tier and high-tier registration time distributions.

These studies, however, only consider voice service but do not consider both voice and data services together. Thus, low tier always has higher priority than high tier, and if both low tier and high tier are available simultaneously, an MT is always registered at the low tier. In the integrated WLAN/UMTS networks, however, the type of service, i.e., voice service and data service, should be considered for tier selection because although low tier WLAN provides higher data rate services, UMTS should be used for voice service in order to meet the QoS requirement of realtime voice service. Thus, previous SR and MR schemes cannot be applied to systems directly considering both voice and data services, and appropriate extensions should be made. 
Since current WLAN network is not appropriate for voice service with real-time service requirement, an MT should not change its network from UMTS to WLAN when the MT with voice service moves to WLAN coverage area. On the other hand, if the MT with data service moves to WLAN coverage area, it should change its network from UMTS to WLAN since WLAN supports higher data service. In this paper, we improve the SR and MR schemes proposed in [7] - [10] by considering both voice and data services together and analyze the performance tradeoff of these schemes.

This paper is organized as follows: Section 2 proposes the improved SR and MR schemes for integrated WLAN/UMTS networks. In Section 3 , the performance of the improved SR and MR schemes is analyzed. Numerical examples are provided in terms of network signaling cost, radio signaling cost, and total signaling cost in Section 4 Finally, conclusions and further studies are presented in Section 5

\section{Improved Registration Schemes for Integrated WLAN/UMTS Networks}

In this paper, we assume a simple WLAN/UMTS network area as shown in Fig. 1 , where WLAN and UMTS networks are fully overlapped and a UMTS RA contains multiple WLAN hotspots. For simplicity, it is assumed that a WLAN hotspot consists of one WLAN RA. In [7] - [10], an MT is allowed to register with MHLR on only one tier in SR scheme and it is allowed to register with MHLR on multiple tiers at the same time in MR scheme. In SR scheme, there are two alternatives, i.e., SR1 and SR2 [7] [10]. In SR1 scheme, the current RA of an MT is stored in MHLR. Suppose that the MT $m$ moves between tiers as shown in Fig. 2, where it is in high tier RA H1 initially (1). In Fig. 2, high tier corresponds to UMTS and low tier corresponds to WLAN. Then, the location information is managed in MHLR as $(m, H 1)$. If the MT moves from high tier RA H1 to RA L1 of low tier (2), then there is registration request from the visitor location registration (VLR) of L1 to the MHLR and cancellation request is sent from the MHLR to the VLR of RA H1. In this case, $(m, L 1)$ is stored in MHLR. If the MT moves from RA L1 to RA H1 (3), registration from the VLR of RA H1 to MHLR and following cancellation from MHLR to the VLR of RA L1 are performed, and ( $m$, H1) is stored in MHLR. Finally, if MT moves from RA H1 to RA L2 of low tier (4),

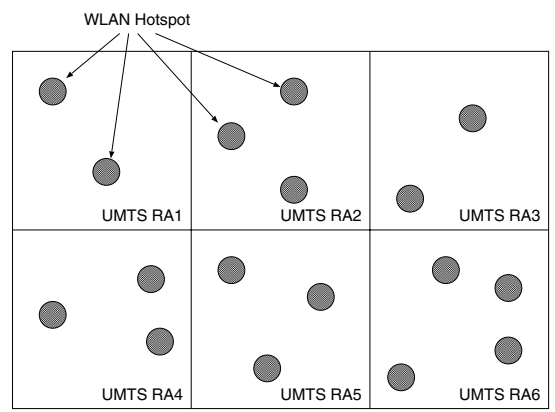

Fig. 1. A WLAN/UMTS network area 


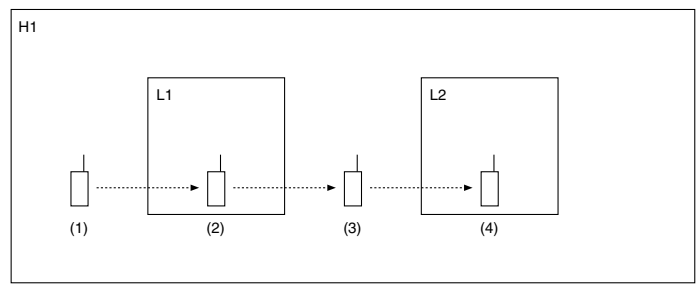

Fig. 2. Movement of MT between different tiers

registration from the VLR of RA L1 to the MHLR and following cancellation from MHLR to the VLR of RA H are performed, and ( $m, \mathrm{~L} 2)$ is stored in MHLR.

In SR2 scheme, MHLR stores location information consisting of two location fields (one for high tier and one for low tier) and one bit to indicate the availability of tier [7] [10]. Suppose that the MT $m$ is in the high tier with RA H1 and the previous low tier RA is L1 (1). Then, location record $(m, \mathrm{H} ; \mathrm{H} 1, \mathrm{~L} 1)$ is stored in the MHLR. If the MT moves from RA H1 to low tier RA L1 (2), the VLR of RA L1 sends tier switching request to MHLR and the MHLR changes the tier bit to L, and the location record $(m, \mathrm{~L} ; \mathrm{H} 1, \mathrm{~L} 1)$ is stored in the MHLR. In this case, there is no cancellation from MHLR to the VLR of RA H1. If the MT moves from RA L1 to RA H1 (3), only tier switching is performed and $(m, \mathrm{H} ; \mathrm{H} 1, \mathrm{~L} 1)$ is stored in the MHLR. Finally, if MT moves from RA H1 to RA L2 of low tier (4), registration and tier switching request are delivered from the VLR of RA L2 to MHLR and cancellation from MHLR to the VLR of L1 is performed. Location record $(m, \mathrm{~L} ; \mathrm{H} 1, \mathrm{~L} 2)$ is stored in MHLR.

In MR scheme, MHLR stores most recently visited low tier RA and high tier RA information [7] - [10]. If the MT $m$ is in H1 and the most recently visited low tier RA is L1 (1), $(m, \mathrm{H} 1, \mathrm{~L} 1)$ is stored in MHLR. If the MT moves to RA L1 (2), there is no registration and cancellation procedure since L1 is the same low tier RA as stored in the MHLR. If the MT moves from RA L1 to RA H1 of high tier (3), there is also no registration and cancellation procedure since $\mathrm{H} 1$ is the same high tier RA as stored in MHLR. Finally, if MT moves to RA L2 of low tier (4), VLR of RA L2 sends registration request to MHLR, cancellation request is sent to the VLR of RA L1 from MHLR, and $(m, \mathrm{H} 1, \mathrm{~L} 2)$ is stored in the MHLR. The location information of SR1, SR2, and MR schemes in MHLR is summarized in Table 1. In these SR1 and SR2 scheme, low tier has higher priority than high tier, and thus, if both low tier and high tier are available simultaneously, MT is always registered at the low tier. This is because although both low tier and high tier network support voice service, low cost is paid for using low tier.

Table 1. Location information of SR1, SR2, and MR schemes in MHLR

\begin{tabular}{|c|c|c|c|c|}
\hline & $(1)$ & $(2)$ & $(3)$ & $(4)$ \\
\hline SR1 & $(\mathrm{m}, \mathrm{H} 1)$ & $(\mathrm{m}, \mathrm{L} 1)$ & $(\mathrm{m}, \mathrm{H} 1)$ & $(\mathrm{m}, \mathrm{L} 2)$ \\
\hline SR2 & $(\mathrm{m}, \mathrm{H} ; \mathrm{H} 1, \mathrm{~L} 1)$ & $(\mathrm{m}, \mathrm{L} ; \mathrm{H} 1, \mathrm{~L} 1)$ & $(\mathrm{m}, \mathrm{H} ; \mathrm{H} 1, \mathrm{~L} 1)$ & $(\mathrm{m}, \mathrm{L}, \mathrm{H} 1, \mathrm{~L} 2)$ \\
\hline MR & $(\mathrm{m}, \mathrm{H} 1, \mathrm{~L} 1)$ & $(\mathrm{m}, \mathrm{H} 1, \mathrm{~L} 1)$ & $(\mathrm{m}, \mathrm{H} 1, \mathrm{~L} 1)$ & $(\mathrm{m}, \mathrm{H} 1, \mathrm{~L} 2)$ \\
\hline
\end{tabular}


In this paper, however, we consider both voice service and data service together in WLAN/UMTS network. Since current WLAN does not satisfy QoS of realtime voice service, higher priority should be given to UMTS network for voice service, which is the major difference from the previous multi-tier location management schemes. On the contrary, WLAN should have higher priority for data service as in the previous schemes. Thus, SR1, SR2, and MR schemes should be improved appropriately in order to satisfy the QoS of both voice and data services in integrated WLAN/UMTS networks. Thus, we improved SR1, SR2, and MR, and they are denoted as ISR1, ISR2, and IMR, respectively.

In this paper, we consider the operation of ISR1, ISR2, and IMR for power on/off, registration, and voice/data call delivery procedures. When the MT is initially turned on, it performs power on registration. In ISR1, the registration request is sent through either WLAN or UMTS based on the network where the MT is located when the MT is turned on. If the MT is inside WLAN coverage area, it performs power on registration through WLAN. On the other hand, if the MT is outside WLAN coverage area, then power on registration is performed only through UMTS. In ISR2, power on registration is performed similarly as in ISR1. In IMR, the MT performs two separate power on registrations through both WLAN and UMTS when the MT is turned on inside WLAN coverage area. If the MT is outside WLAN coverage area, power on registration is performed only through UMTS.

When the MT is turned off, power off deregistration is performed. In ISR1, if the MT is inside WLAN when the MT is turned off, it only performs power off deregistration through WLAN network. On the other hand, in ISR2 and IMR, if the deregistration message is sent to MHLR via WLAN, additional deregistration message is sent from MHLR to UMTS. Likewise, if the deregistration message is sent to MHLR via UMTS, additional deregistration message is sent from MHLR to WLAN.

The number of network signaling and radio signaling messages for power on registration and power off deregistration is summarized in Table 2, where tier denotes at which tier an MT is located when the power on registration and power off deregistration events occur, and system denotes at which system either network or radio signaling messages occur.

Table 3 summarizes network signaling and radio signaling messages for registration by movement of MT, where $P_{s w}$ is the probability that the WLAN network visited by MT is the same WLAN network that the MT visited most recently and direction means the direction of movement by the MT. In ISR1, there are always two signaling messages in both WLAN and UMTS for the movement in both directions. Note that there is no signaling message for a transition from low tier to high tier in IMR scheme. The number of messages can be obtained based on the operations of ISR1, ISR2, and IMR schemes.

Likewise, Table 4 summarizes the number of network signaling and radio signaling messages for voice/data call delivery, where $N_{\text {cell }}$ denotes the number of cells in an RA in UMTS networks. We note that a WLAN RA is assumed to consist of single WLAN cell. In ISR1 and ISR2, voice/data calls should be delivered to the currently registered network irrespective the type of call. On the other hand, in IMR, voice call is always delivered through UMTS because WLAN is not appropriate for satisfying the QoS of voice service. For data call, since WLAN has higher priority than UMTS, it is firstly delivered through WLAN and if it fails, it is delivered to UMTS. 
Table 2. Number of network signaling and radio signaling message for power on registration and power off deregistration

\begin{tabular}{|c|c|c|c|c|c|c|}
\hline \multirow{2}{*}{ Scheme } & \multirow{2}{*}{ Tier } & \multirow{2}{*}{ System } & \multicolumn{2}{|c|}{ Power on } & \multicolumn{2}{|c|}{ Power off } \\
\hline & & & \begin{tabular}{|l|} 
Network \\
\end{tabular} & Radio & Network & $\overline{\text { Radio }}$ \\
\hline \multirow{4}{*}{ ISR1 } & \multirow{2}{*}{ Low } & WLAN & 2 & 2 & 2 & 2 \\
\hline & & UMTS & 0 & 0 & 0 & 0 \\
\hline & \multirow{2}{*}{ High } & WLAN & 0 & 0 & 0 & 0 \\
\hline & & UMTS & 2 & 2 & 2 & 2 \\
\hline \multirow{4}{*}{ ISR2 } & \multirow{2}{*}{ Low } & WLAN & 2 & 2 & 2 & 2 \\
\hline & & UMTS & 0 & 0 & 2 & 0 \\
\hline & \multirow{2}{*}{ High } & WLAN & 0 & 0 & 2 & 0 \\
\hline & & UMTS & 2 & 2 & 2 & 2 \\
\hline \multirow{4}{*}{ IMR } & \multirow[b]{2}{*}{ Low } & WLAN & 2 & 2 & 2 & 2 \\
\hline & & UMTS & 2 & 2 & 2 & 0 \\
\hline & \multirow{2}{*}{ High } & WLAN & 0 & 0 & 2 & 2 \\
\hline & & UMTS & 2 & 2 & 2 & 0 \\
\hline
\end{tabular}

Table 3. Number of network signaling and radio signaling message for registration by movement of MT

\begin{tabular}{|c|c|c|c|c|}
\hline \multirow{2}{*}{ Scheme } & \multirow{2}{*}{ Direction } & \multirow{2}{*}{ System } & \multicolumn{2}{|l|}{ Registration } \\
\hline & & & Network & Radio \\
\hline \multirow{4}{*}{ ISR1 } & \multirow{2}{*}{ High $\rightarrow$ Low } & WLAN & 2 & 2 \\
\hline & & UMTS & 2 & 0 \\
\hline & \multirow{2}{*}{ Low $\rightarrow$ High } & WLAN & 2 & 0 \\
\hline & & UMTS & 2 & 2 \\
\hline \multirow{4}{*}{ ISR2 } & \multirow{2}{*}{ High $\rightarrow$ Low } & WLAN & $2 \mathrm{P}_{s w}+4\left(1-\mathrm{P}_{s w}\right)$ & $2 \mathrm{P}_{s w}+2\left(1-\mathrm{P}_{s w}\right)$ \\
\hline & & UMTS & 0 & 0 \\
\hline & \multirow{2}{*}{ Low $\rightarrow$ High } & WLAN & 0 & 0 \\
\hline & & UMTS & 2 & 2 \\
\hline \multirow{4}{*}{ IMR } & \multirow{2}{*}{ High $\rightarrow$ Low } & WLAN & $4\left(1-\mathrm{P}_{s w}\right)$ & $2\left(1-\mathrm{P}_{s w}\right)$ \\
\hline & & UMTS & 0 & 0 \\
\hline & \multirow{2}{*}{ Low $\rightarrow$ High } & WLAN & 0 & 0 \\
\hline & & UMTS & 0 & 0 \\
\hline
\end{tabular}

\section{Performance Analysis}

For performance analysis, we make the following assumptions:

- Incoming voice call arrival and data call arrival at an MT occur according to a Poisson process with parameters $\lambda_{V}$ and $\lambda_{D}$, respectively;

- WLAN residence time follows a general distribution with mean $1 / \mu_{w}$;

- The interval from the time that an MT moves out of a WLAN coverage area to the time that the MT moves into next WLAN coverage area follows a general distribution with mean $1 / \mu_{u}$. 
Table 4. Number of network signaling and radio signaling message for voice/data call delivery

\begin{tabular}{|c|c|c|c|c|c|c|}
\hline \multirow{2}{*}{ Scheme } & \multirow{2}{*}{ Tier } & \multirow{2}{*}{ System } & \multicolumn{2}{|c|}{ Voice call } & \multicolumn{2}{|l|}{ Data call } \\
\hline & & & $\mid \overline{\text { Network }}$ & Radio & Network & Radio \\
\hline \multirow{4}{*}{ ISR1 } & \multirow{2}{*}{ Low } & WLAN & 2 & 2 & 2 & 2 \\
\hline & & UMTS & 0 & 0 & 0 & 0 \\
\hline & \multirow{2}{*}{ High } & WLAN & 0 & 0 & 0 & 0 \\
\hline & & UMTS & 2 & $\mathrm{~N}_{\text {cell }}+1$ & 2 & $\mathrm{~N}_{\text {cell }}+1$ \\
\hline \multirow{4}{*}{ ISR2 } & \multirow{2}{*}{ Low } & WLAN & 2 & 2 & 2 & 2 \\
\hline & & UMTS & 0 & 0 & 0 & 0 \\
\hline & \multirow{2}{*}{ High } & WLAN & 0 & 0 & 0 & 0 \\
\hline & & UMTS & 2 & $\overline{\mathrm{N}_{\text {cell }}+1}$ & 2 & $\mathrm{~N}_{\text {cell }}+1$ \\
\hline \multirow{4}{*}{ IMR } & \multirow{2}{*}{ Low } & WLAN & 0 & 0 & 2 & 2 \\
\hline & & UMTS & 2 & $\mathrm{~N}_{\text {cell }}+1$ & 0 & 0 \\
\hline & \multirow{2}{*}{ High } & WLAN & 0 & 0 & 0 & 0 \\
\hline & & UMTS & 2 & $\widehat{\mathrm{N}_{\text {cell }}+1}$ & 2 & $\widehat{\mathrm{N}_{\text {cell }}+1}$ \\
\hline
\end{tabular}

In order to analyze the performance of ISR1, ISR2, and IMR, unit costs for network signaling load and radio signaling load are defined. The $h_{w}^{r e g}, h_{w}^{p a g}, h_{u}^{r e g}$, and $h_{u}^{p a g}$ are defined as the unit network signaling cost for registration in WLAN, paging in WLAN, registration in UMTS, and paging in UMTS, respectively. The $g_{w}^{r e g}, g_{w}^{\text {Vpag }}$, $g_{w}^{D p a g}, g_{u}^{r e g}, g_{u}^{V p a g}$, and $g_{u}^{D p a g}$ are defined as the unit radio signaling cost for registration in WLAN, paging for voice service in WLAN, paging for data service in WLAN, registration in UMTS, paging for voice service in UMTS, and paging for data service in UMTS, respectively.

Using the unit costs, network and radio signaling costs for power on registration for ISR1, ISR2, and IMR schemes are obtained based on Table 2 as follows:

$$
\begin{aligned}
N C_{I S R 1}^{o n} & =2 P_{w} h_{w}^{r e g}+2 P_{u} h_{u}^{r e g}, \\
N C_{I S R 2}^{o n} & =2 P_{w} h_{w}^{r e g}+2 P_{u} h_{u}^{r e g}, \\
N C_{I M R}^{o n} & =P_{w}\left(2 h_{w}^{r e g}+2 h_{u}^{r e g}\right)+2 P_{u} h_{u}^{r e g}, \\
R C_{I S R 1}^{o n} & =2 P_{w} g_{w}^{r e g}+2 P_{u} g_{u}^{r e g} \\
R C_{I S R 2}^{o n} & =2 P_{w} g_{w}^{r e g}+2 P_{u} g_{u}^{r e g}, \\
R C_{I M R}^{o n} & =P_{w}\left(2 g_{w}^{r e g}+2 g_{u}^{r e g}\right)+2 P_{u} g_{u}^{r e g},
\end{aligned}
$$

where $P_{w}$ and $P_{u}$ represent the probability that the MT is inside WLAN coverage and outside WLAN coverage when the MT is initially turned on and they are given as follows:

$$
P_{w}=\frac{\mu_{u}}{\mu_{w}+\mu_{u}}, \quad P_{u}=\frac{\mu_{w}}{\mu_{w}+\mu_{u}} .
$$

Likewise, network and radio signaling costs for power off deregistration for ISR1, ISR2, and IMR schemes are obtained based on Table 2 as follows: 


$$
\begin{aligned}
N C_{I S R 1}^{o f f} & =2 P_{w} h_{w}^{r e g}+2 P_{u} h_{u}^{r e g} \\
N C_{I S R 2}^{o f f} & =2 P_{w}\left(2 h_{w}^{r e g}+2 h_{u}^{r e g}\right)+P_{u}\left(2 h_{w}^{r e g}+2 h_{u}^{r e g}\right), \\
N C_{I M R}^{o f f} & =P_{w}\left(2 h_{w}^{r e g}+2 h_{u}^{r e g}\right)+P_{u}\left(2 h_{w}^{r e g}+2 h_{u}^{r e g}\right) \\
R C_{I S R 1}^{o f f} & =2 P_{w} g_{w}^{r e g}+2 P_{u} g_{u}^{r e g} \\
R C_{I S R 2}^{o f f} & =2 P_{w} g_{w}^{r e g}+2 P_{u} g_{u}^{r e g} \\
R C_{I M R}^{o f f} & =2 P_{w} g_{w}^{r e g}+2 P_{u} g_{u}^{r e g}
\end{aligned}
$$

Network and radio signaling costs for registration for ISR1, ISR2, and IMR schemes are obtained based on Table 3 as follows:

$$
\begin{aligned}
N C_{I S R 1}^{r e g} & =2 h_{w}^{r e g}+2 h_{u}^{r e g}+h_{w}^{r e g}+2 h_{u}^{r e g}, \\
N C_{I S R 2}^{r e g} & =h_{w}^{r e g}\left(2 P_{s w}+4\left(1-P_{s w}\right)\right)+2 h_{u}^{r e g}, \\
N C_{I M R}^{r e g} & =4 h_{w}^{r e g}\left(1-P_{s w}\right), \\
R C_{I S R 1}^{r e g} & =2 g_{w}^{r e g}+2 g_{u}^{r e g}, \\
R C_{I S R 2}^{r e g} & =g_{w}^{r e g}\left(2 P_{s w}+2\left(1-P_{s w}\right)\right)+2 g_{u}^{r e g}, \\
R C_{I M R}^{r e g} & =2 g_{w}^{r e g}\left(1-P_{s w}\right) .
\end{aligned}
$$

Network and radio signaling costs for voice call delivery for ISR1, ISR2, and IMR schemes are obtained based on Table 4 as follows:

$$
\begin{aligned}
& N C_{I S R 1}^{V p a g}=2 P_{w} h_{w}^{p a g}+2 P_{u} h_{u}^{p a g}, \\
& N C_{I S R 2}^{V p a g}=2 P_{w} h_{w}^{p a g}+2 P_{u} h_{u}^{p a g}, \\
& N C_{I M R}^{V p a g}=2 P_{w} h_{u}^{p a g}+2 P_{u} h_{u}^{p a g}, \\
& R C_{I S R 1}^{V p a g}=2 P_{w} g_{w}^{V p a g}+P_{u}\left(N_{\text {cell }}+1\right) g_{u}^{V p a g}, \\
& R C_{I S R 2}^{V p a g}=2 P_{w} g_{w}^{V p a g}+P_{u}\left(N_{\text {cell }}+1\right) g_{u}^{V p a g}, \\
& R C_{I M R}^{V p a g}=P_{w}\left(N_{\text {cell }}+1\right) g_{u}^{V p a g}+P_{u}\left(N_{\text {cell }}+1\right) g_{u}^{V p a g} .
\end{aligned}
$$

Finally, network and radio signaling costs for data call delivery for ISR1, ISR2, and IMR schemes are obtained based on Table 4 as follows:

$$
\begin{aligned}
N C_{I S R 1}^{\text {Dpag }} & =2 P_{w} h_{w}^{\text {pag }}+2 P_{u} h_{u}^{\text {pag }} \\
N C_{I S R 2}^{\text {Dpag }} & =2 P_{w} h_{w}^{\text {pag}}+2 P_{u} h_{u}^{\text {pag }} \\
N C_{I M R}^{\text {Dpag }} & =2 P_{w} h_{u}^{\text {pag }}+2 P_{u} h_{u}^{\text {pag }} \\
R C_{I S R 1}^{\text {Dpag }} & =2 P_{w} g_{w}^{\text {Dpag }}+P_{u}\left(N_{c e l l}+1\right) g_{u}^{\text {Dpag }}, \\
R C_{I S R 2}^{\text {Dpag }} & =2 P_{w} g_{w}^{\text {Dpag }}+P_{u}\left(N_{c e l l}+1\right) g_{u}^{\text {Dpag }}, \\
R C_{I M R}^{\text {Dpag }} & =2 P_{w} g_{w}^{\text {Dpag }}+P_{u}\left(g_{w}^{\text {Dpag }}+\left(N_{\text {cell }}+1\right) g_{u}^{\text {Dpag }}\right) .
\end{aligned}
$$


Based on the above results, total expected network and radio signaling costs for each scheme are derived as follows:

$$
\begin{aligned}
N C_{i} & =N C_{i}^{o n}+N C_{i}^{\text {fff }}+N C_{i}^{\text {reg }} N_{\text {cycle }} \\
& +\left(N C_{i}^{\text {Vpag }} \lambda_{V}\left(1 / \mu_{w}+1 / \mu_{u}\right)\right. \\
& \left.+N C_{i}^{\text {Dpag }} \lambda_{D}\left(1 / \mu_{w}+1 / \mu_{u}\right)\right) N_{\text {cycle }}, \\
R C_{i} & =R C_{i}^{\text {on }}+R C_{i}^{o f f}+R C_{i}^{\text {reg }} N_{\text {cycle }} \\
& +\left(R C_{i}^{\text {Vpag }} \lambda_{V}\left(1 / \mu_{w}+1 / \mu_{u}\right)\right. \\
& \left.+R C_{i}^{\text {Dpag }} \lambda_{D}\left(1 / \mu_{w}+1 / \mu_{u}\right)\right) N_{\text {cycle }},
\end{aligned}
$$

where $i$ denotes each location registration scheme, i.e., ISR1, ISR2, and IMR, and $N_{\text {cycle }}$ is the total expected number of the movement into or out of WLAN coverage area during power on period, which is obtained by $\frac{1 / \mu_{a}}{1 / \mu_{u}+1 / \mu_{w}}$. Finally, total signaling cost for each scheme is defined as follows:

$$
C_{i}=w_{n e t} N C_{i}+w_{\text {rad }} R C_{i}
$$

where $w_{\text {net }}$ and $w_{\text {rad }}$ are weighting factors for network signaling cost and radio signaling cost, respectively since these two costs are not directly comparable.

\section{Numerical Examples}

In this section, numerical examples are given in order to investigate the tradeoff among the ISR1, ISR2, and IMR. Default parameter values for network and radio signaling cost, and mobility and traffic characteristics are summarized in Tables 5 and 6, respectively. In Table 5, we give higher values for unit radio signaling cost for registration than that for paging because it is widely accepted that more data bytes are needed for registration and thus, consumes more radio bandwidth.

Figs. 3 (a), (b), and (c) show radio, network, and total signaling cost for varying the values of $1 / \mu_{w}$. As can be seen in Fig. 3 (a), ISR1 and ISR2 have the same radio signaling cost, as expected. For small values of $1 / \mu_{w}$, i.e., frequent movements out of WLAN coverage area, IMR performs better than ISR1 and ISR2 due to less registration

Table 5. Default parameter values for unit network and radio signaling costs

\begin{tabular}{|c|c|c|c|c|c|c|c|c|c|c|}
\hline$h_{w}^{\text {reg }}$ & $h_{w}^{\text {pag }}$ & $h_{u}^{\text {reg }}$ & $h_{u}^{\text {pag }}$ & $g_{w}^{\text {reg }}$ & $g_{w}^{\text {Vag }}$ & $g_{w}^{\text {Dag }}$ & $g_{u}^{\text {reg }}$ & $g_{u}^{\text {Vag }}$ & $g_{u}^{\text {Dag }}$ \\
\hline \hline 1 & 1 & 1 & 1 & 5 & 1 & 1 & 5 & 1 & 1 \\
\hline
\end{tabular}

Table 6. Default parameter values for mobility and traffic characteristics

\begin{tabular}{|c|c|c|c|c|c|c|c|c|c|}
\hline$\lambda_{V}$ & $\lambda_{D}$ & $\mu_{a}$ & $\mu_{w}$ & $\mu_{u}$ & $P_{s w}$ & $N_{\text {cycle }}$ & $N_{\text {cell }}$ & $w_{\text {net }}$ & $w_{\text {rad }}$ \\
\hline \hline 1.5 & 10 & 0.1 & 10 & 1 & 0.1 & 20 & 20 & 1 & 5 \\
\hline
\end{tabular}




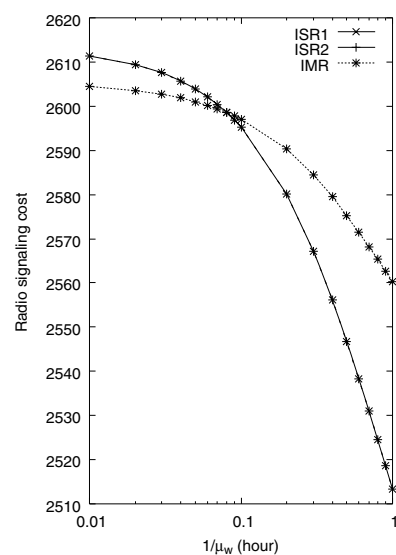

(a) Radio signaling cost

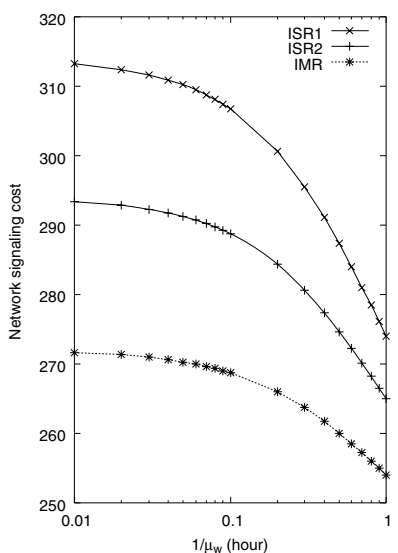

(b) Network signaling cost

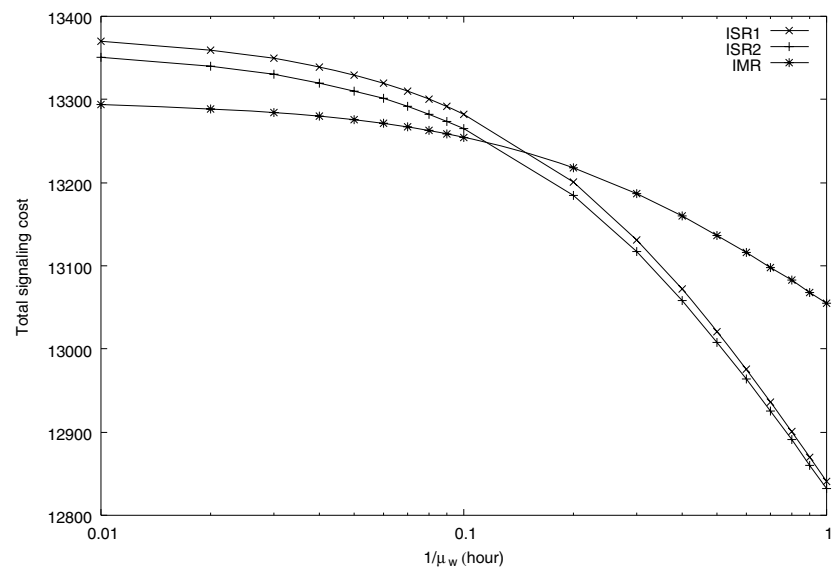

(c)Total signaling cost

Fig. 3. Signaling cost for varying $1 / \mu_{w}$

signaling cost. For large values of $1 / \mu_{w}$, on the other hand, ISR1 and ISR2 perform better than IMR due to less paging signaling cost. Since the number of tier switching is the smallest in IMR, network signaling cost of IMR is the smallest. On the other hand, the network signaling cost of ISR1 is the largest due to the largest number of tier switching. It can be seen that ISR2 performs better than ISR1 for the considered parameter sets and there is tradeoff between IMR and others.

Fig. 4 shows total signaling for varying the values of $P_{s w}$. As can be expected, the total signaling cost of IMR decreases as the value of $P_{s w}$ increases because if $P_{s w}$ is high, the probability of tier switching in IMR is small. We note that the total signaling cost of ISR1 does not vary for the change of $P_{s w}$ because new registration at new tier and new deregistration at old tier always occur due to the movement between low tiers and high tiers by MT. There is also tradeoff among the three schemes. 


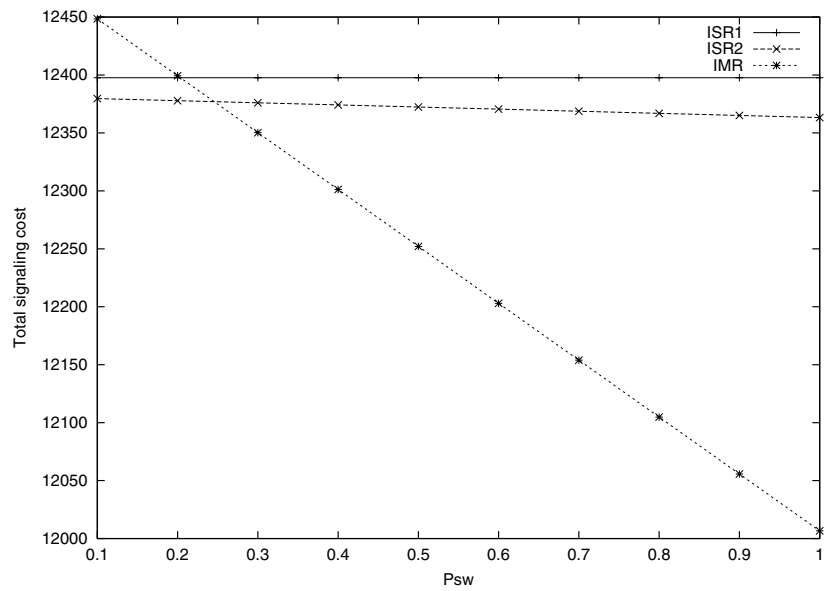

Fig. 4. Total signaling cost for varying $P_{s w}$

\section{Conclusions and Further Studies}

In this paper, conventional SR and MR schemes are improved appropriately in order to accommodate both voice and data services appropriately in integrated WLAN/UMTS networks. The number of network signaling and radio signaling messages for power on registration, power off deregistration, registration by movement of MT, and voice/data call delivery is obtained. Then, the performance of ISR1, ISR2, and IMR schemes is analyzed based on mobility and traffic characteristics of mobile users in terms of radio, network, and total signaling costs. The numerical results investigate the performance variation of the proposed schemes for varying the characteristics parameter. It is concluded that there is tradeoff among the three schemes and thus, an appropriate scheme should be selected based on mobility and traffic characteristics. The analytical methodology developed in this paper can be extended to analyze the performance of general heterogeneous networks with multiple wireless access systems, which will be realized in $4 \mathrm{G}$ networks. As further studies, we are doing research on the following topics:

- More realistic modelling of integrated WLAN/UMTS network residence time distributions;

- More accurate analysis of network, radio, and total costs based on more detailed modelling of mobility and traffic characteristics of mobile users;

- Dynamic selection of multi-tier location management schemes based on mobile user's varying mobility and traffic characteristics.

These further studies can help to solve the location management problem in $4 \mathrm{G}$ heterogeneous networks with multiple wireless access systems such as GSM, UMTS, WLAN, Satellite, and DVB, with the aid of the analytical methodology developed in this paper.

\section{Acknowledgement}

This work was supported by the Soongsil University Research Fund. 


\section{References}

1. I. F. Akyildiz, J. Xie, and S. Mohanty, "A survey of mobility management in next-generation all-IP-based wireless systems," IEEE Wireless Communications, pp. 16-28, Aug. 2004.

2. 3GPP TR 22.934 V6.2.0, "Feasibility study on 3GPP system to wireless local area network (WLAN) interworking," Sep. 2003.

3. 3GPP TR 23.234, "3GPP system to wireless local area network (WLAN) interworking; system description," Jan. 2004.

4. M. Jaseemuddin, "Architecture for integrating UMTS and 802.11 WLAN networks," IEEE International Symposium on Computers and Communication, pp. 2003.

5. V. K. Varma, S. Ramesh, K. D. Wong, M. Barton, G. Hayward, and J. A. Friedhoffer, "Mobility management in integrated UMTS/WLAN networks," IEEE International Conference on Communications, pp. 1048-1053, 2003.

6. B. Sarikaya and T. Ozugur, "Dormant mode operation support for roaming from WLAN to UMTS," IEEE International Conference on Communications, pp. 1038-1042, 2003.

7. Y. B. Lin and I. Chlamtac, "Heterogeneous personal communications services: integration of PCS systems," IEEE Communications Magazine, vol. 3, no. 9, pp.106-112, Sep. 1996.

8. Y. B. Lin, "A comparison study of the two-tier and the single-tier personal communications service systems," ACM/Baltzer Mobile Networks and Applications, vol. 1, no. 1, pp. 29-38, 1996.

9. Y. Fang, "Registration traffic and service availability for two-tier wireless networks," IEEE Wireless Communications and Networking Conference, pp. 1090-1095, 2000.

10. Y. Fang and Y.B. Lin, "Mobility management and signaling traffic analysis for multi-tier wireless mobile metworks," IEEE Transactions on Vehicular Technology, vol. 54, no. 5, pp. 1843-1853, Sept. 2005 